\title{
DYNAMICAL SYSTEMS WITH AN INVARIANT SPACE OF VECTOR FIELDS
}

\author{
BY \\ ROBERT I. JEWETT AND SOL SCHWARTZMAN
}

I. In the theory of differentiable dynamical systems, the natural actions on coset spaces are of particular interest, not least because the general situation is so intractable. Let $G$ be a Lie group and $H$ a closed subgroup such that $G / H$ is compact. A natural action on $G / H$ is a group of transformations of $G / H$ of the form $x H \mapsto a \alpha(x) H$, where $a$ is an element of $G$ and $\alpha$ is an automorphism of $G$ such that $\alpha(H)=H$. The space of right-invariant vector fields on $G$ is carried by the natural projection $\pi, x \mapsto x H$, onto a space of vector fields on $G / H$, and this space is invariant under each natural action. Suppose now that $H$ is discrete. Then $G$ is unimodular. A Haar measure on $G$ determines a finite Borel measure on $G / H$ invariant under each action. Analogously, a translation invariant $n$-form $\omega$ on $G$, where $n$ is the dimension of $G$, determines an $n$-form $\eta$ on $G / H$, and $T^{*} \eta$ equals $\pm \eta$ for each natural transformation $T$.

It is such a phenomenon that we examine in this paper: A dynamical system in which a certain finite-dimensional linear space of vector fields and a certain differential form are (essentially) invariant under the action.

In the next few paragraphs we define several expressions, and then state our main result. The following section is devoted to a proof of this theorem. Additional results are presented in the third section.

Let $M$ be a differentiable manifold and let $\mathscr{X}$ be a finite-dimensional linear space of vector fields on $M$. We say that $\mathscr{X}$ is spanning if, for each $p$ in $M$, the evaluation at $p, X \mapsto X_{p}$, maps $\mathscr{X}$ onto the tangent space of $M$ at $p$. If the dimension of $\mathscr{X}$ equals the dimension of $M$, in which case these mappings are each one-to-one, then $\mathscr{X}$ is said to be simply spanning. If $\mathscr{X}$ is simply spanning, an affine connection $\nabla$ on $M$ is determined by the condition:

$$
\nabla_{Y} X=0
$$

for $X$ in $\mathscr{X}$ and $Y$ any vector field on $M$. We call $\nabla$ the connection associated with $\mathscr{X}$.

Let $\mathscr{T}$ be a group of diffeomorphisms of $M$. For each $T$ in $\mathscr{T}$, let $T_{*}$ be the associated mapping of vector fields, and let $T^{*}$ be the associated mapping of differential forms. A linear space $\mathscr{X}$ of vector fields on $M$ is said to be invariant under $\mathscr{T}$, if $T_{*}(\mathscr{X})=\mathscr{X}$ for each $T$ in $\mathscr{T}$. In this case, the restriction of $\left\{T_{*}: T \in \mathscr{T}\right\}$ to $\mathscr{X}$ is a group of linear transformations of $\mathscr{X}$.

Received by the editors February 21, 1969.

Copyright (C) 1969, American Mathematical Society 
Let $\mathscr{T}$ be a group of homeomorphisms of a space $S$. An eigenfunction for $\mathscr{T}$ on $S$ is a function $f$ on $S$, with values in the unit circle, such that, for each $T$ in $\mathscr{T}$, there exists a complex number $\lambda_{T}$ satisfying $f T=\lambda_{T} f$.

THEOREM 1. Let $M$ be a compact differentiable manifold of dimension $n, \omega$ an $n$-form on $M, \mathscr{X}$ a finite-dimensional linear space of vector fields on $M$, and $\mathscr{T} a$ group of diffeomorphisms of $M$. Suppose the following statements are true.

(I) $\omega$ is not identically zero, and $T^{*} \omega= \pm \omega$ for each $T$ in $\mathscr{T}$.

(II) $\mathscr{X}$ is spanning and invariant under $\mathscr{T}$.

(III) $\mathscr{T}$ is abelian and each differentiable eigenfunction for $\mathscr{T}$ on $M$ is constant.

Then the following statements are also true.

(I) Except for multiplication by a scalar, $\omega$ is the only $n$-form on $M$ satisfying assumption (I) above.

(II) $\mathscr{X}$ is simply spanning.

(III) If $\mathscr{Y}$ is a finite-dimensional linear space of vector fields on $M$ invariant under $\mathscr{T}$, then $\mathscr{Y} \subseteq \mathscr{X}$.

(IV) $\mathscr{X}$ is a Lie algebra.

(V) The system $(M, \omega, \mathscr{X}, \mathscr{T})$ is isomorphic to $(G / \Gamma, \eta, \mathscr{Y}, \mathscr{S})$ where: $G$ is a simply connected Lie group whose Lie algebra is isomorphic to $\mathscr{X} ; \Gamma$ is a discrete subgroup of $G ; \eta$ corresponds to a translation-invariant $n$-form on $G ; \mathscr{Y}$ corresponds to the space of right-invariant vector fields on $G$; and, each transformation, $x \Gamma \mapsto S(x \Gamma)$, in $\mathscr{S}$ is of the form

$$
x \Gamma \mapsto a \alpha(x) \Gamma,
$$

a being in $G$ and $\alpha$ being an automorphism of $G$ such that $\alpha(\Gamma)=\Gamma$.

COROLlaRY 1. Let $M$ be a compact differentiable manifold, $\nabla$ an affine connection on $M$ associated with a simply spanning space of vector fields, and $\mathscr{T}$ an abelian group of diffeomorphisms of $M$ which preserve the affine structure. If each differentiable eigenfunction for $\mathscr{T}$ on $M$ is constant, then $\mathscr{T}$ preserves no other such affine structure, and $(M, \mathscr{T})$ is isomorphic to a natural action on a coset space $G / \Gamma$ with $\Gamma$ discrete.

II.

LEMMA 1. Let $\mathscr{E}$ be a finite-dimensional real vector space, $\mathscr{L}$ an abelian group of linear transformations of $\mathscr{E}$, and $C$ a compact subset of $\mathscr{E}$ invariant under $\mathscr{L}$. If $C$ has more than one point, then there exists a differentiable function $f: \mathscr{E} \rightarrow C$ such that $f$ is an eigenfunction for $\mathscr{L}$ on $C$.

Proof. Let $\mathscr{F}$ be the space of all complex-valued real-linear functionals on $\mathscr{E}$; $\mathscr{F}$ is a complex vector space. For each $L$ in $\mathscr{L}$ define $L^{*}: \mathscr{F} \rightarrow \mathscr{F}$ by $L^{*} f=f L$. Thus, $\mathscr{L}^{*}=\left\{L^{*}: L \in \mathscr{L}\right\}$ is an abelian group of linear transformations of $\mathscr{F}$. These transformations can be put simultaneously in triangular form. That is, there exists a basis $\left\{f_{1}, f_{2}, \ldots, f_{n}\right\}$ of $\mathscr{F}$ such that for each $k$, the linear span of $\left\{f_{1}, \ldots, f_{k}\right\}$ 
is invariant under $\mathscr{L}^{*}$. Let $m$ be the smallest integer $k$ such that $f_{k}$ is not constant on $C$. Set $g=f_{m}$. Hence, for each $L$ in $\mathscr{L}$, there exists $\alpha_{L}$ in $C$ such that $L^{*} g-\alpha_{L} g$ is constant on $C$; let the constant be $\beta_{L}$; on $C$ then, $g L=\alpha_{L} g+\beta_{L}$.

Let $B=g(C)$. Define $A_{L}: C \rightarrow C$ by $A_{L} z=\alpha_{L} z+\beta_{L}$. Since $A_{L}(B)=B,\left|\alpha_{L}\right|=1$. Let $K$ be the convex hull of $C$; then $A_{L}(K)=K$. Thus there exists a point $\gamma$ in $K$ left fixed by each $A_{L}$ :

$$
\gamma=\alpha_{L} \gamma+\beta_{L}
$$

for $L$ in $\mathscr{L}$. Define $h: \mathscr{E} \rightarrow \mathscr{E}$ by $h(x)=g(x)-\gamma$. For $x$ in $C$,

$$
h(L x)=\alpha_{L} g(x)+\beta_{L}-\gamma=\alpha_{L} h(x) .
$$

Thus $h L=\alpha_{L} h$ on $C$ for each $L$.

If $|h|$ is constant on $C$, let the constant be $c$ (which cannot equal 0 ) and define $f$ by $f=h / c$.

If $|h|$ is not constant on $C$, there exists an $\varepsilon>0$ such that

$$
f=e^{i \varepsilon|h|}
$$

is not constant on $C$, and this $f$ is an eigenfunction for $\mathscr{L}$ on $C$, each eigenvalue being 1 in this case.

LeMma 2. Given the assumptions of Theorem 1, conditions (I) and (II) are valid.

Proof. Let $\mathscr{E}$ be the space of all multilinear alternating mappings $\eta: \mathscr{X}^{n} \rightarrow \boldsymbol{R}$; here $\mathscr{X}$ is regarded as a real vector space. Thus $\mathscr{E}$ is a finite-dimensional real vector space. For each $T$ in $\mathscr{T}$, define $T^{\prime}: \mathscr{E} \rightarrow \mathscr{E}$ by

$$
\left(T^{\prime} \eta\right)\left(X_{1}, \ldots, X_{n}\right)=\eta\left(T_{*} X_{1}, \ldots, T_{*} X_{n}\right) .
$$

Each $T^{\prime}$ is linear. For each $T$, let $\varepsilon_{T}$ in $\{1,-1\}$ be such that $T^{*} \omega=\varepsilon_{T} \omega$. Thus

$$
\omega\left(T_{*} X_{1}, \ldots, T_{*} X_{n}\right)=\varepsilon_{T} \omega\left(X_{1}, \ldots, X_{n}\right) T .
$$

For each $p$ in $M$, define $\omega_{p}: \mathscr{X}^{n} \rightarrow \boldsymbol{R}$ by

$$
\omega_{p}\left(X_{1}, \ldots, X_{n}\right)=\left(\omega\left(X_{1}, \ldots, X_{n}\right)\right)(p) .
$$

Clearly $\omega_{p}$ is in $\mathscr{E}$ and

$$
T^{\prime} \omega_{p}=\varepsilon_{T} \omega_{T p}
$$

Define $L_{T}=\varepsilon_{T} T^{\prime}$. Thus $\mathscr{L}=\left\{L_{T}: T \in \mathscr{T}\right\}$ is an abelian group of linear transformations of $\mathscr{E}$ leaving the set

$$
C=\left\{\omega_{p}: p \in M\right\}
$$

invariant. The mapping $p \mapsto \omega_{p}$ of $M$ into $\mathscr{E}$ is differentiable. By Lemma 1 and assumption (III), $C$ has but one point and it is not 0 . 
Conclusion (I) is valid because we have proved that $\omega$ is everywhere nonzero.

To verify (II), suppose that $X$ is in $\mathscr{X}, p$ is in $M$, and $X_{p}=0$. Then

$$
\omega_{p}\left(X, X_{2}, \ldots, X_{n}\right)=0
$$

for $X_{2}, \ldots, X_{n}$ in $\mathscr{X}$. Thus, for each $q$ in $M$,

$$
\omega_{q}\left(X, X_{2}, \ldots, X_{n}\right)=0
$$

for $X_{2}, \ldots, X_{n}$ in $\mathscr{X} ; X_{q}=0$ for each $q ; X=0$.

Lemma 3. Let $\mathscr{X}$ be simply spanning on the compact differentiable manifold $M$ of dimension $n$, and invariant under the group $\mathscr{T}$. Then $M$ is orientable and there exists an everywhere nonzero $n$-form $\omega$ on $M$ with the following property: For each $T$ in $\mathscr{T}$, $T^{*} \omega= \pm \omega$ and the determinant of $T_{*}$ on $\mathscr{X}$ equals \pm 1 , according as (for both) $T$ preserves or reverses the orientation of $M$.

Proof. Let $\left\{X_{1}, X_{2}, \ldots, X_{n}\right\}$ be a basis for $\mathscr{X}$. Let $\left\{\omega_{1}, \omega_{2}, \ldots, \omega_{n}\right\}$ be a dual system of 1-forms on $M$. That is, $\omega_{i}\left(X_{j}\right)=\delta_{i j}$. Set $\omega=\omega_{1} \wedge \omega_{2} \wedge \cdots \wedge \omega_{n}$. For each $T$ in $\mathscr{T}, T^{*} \omega=d_{T} \omega$ where $d_{T}$ is the determinant of $T_{*}$ on $\mathscr{X}$. The finite nonnegative Borel measure on $M$ corresponding to $\omega$ is invariant under $\mathscr{T}$. Hence $\left|d_{T}\right|=1$.

Lemma 4. Let $\mathscr{X}$ be simply spanning on the differentiable manifold $M$. Then there exists a unique affine connection $\nabla$ on $M$ such that $\nabla_{Y} X=0$ for $X$ in $\mathscr{X}$ and $Y$ any vector field on $M$. Moreover, a diffeomorphism $T$ of $M$ is affine with respect to $\nabla$ if and only if $T_{*}(\mathscr{X})=\mathscr{X}$.

Proof. The connection $\nabla$ is given by

$$
\nabla_{Y}\left(\sum_{k=1}^{n} f_{k} X_{k}\right)=\sum_{k=1}^{n}\left(Y f_{k}\right) X_{k}
$$

where $\left\{X_{1}, X_{2}, \ldots, X_{n}\right\}$ is a basis for $\mathscr{X}$. Uniqueness is clear.

Let $T$ be an affine transformation of $M$. Thus $T$ carries geodesics to geodesics, and the geodesics are just the integral curves for members of $\mathscr{X}$. Hence $T_{*}(\mathscr{X})=\mathscr{X}$.

Let $T$ be a diffeomorphism such that $T_{*}(\mathscr{X})=\mathscr{X}$. Let $\nabla^{\prime}$ be the connection on $M$ induced by $T$. That is,

$$
\nabla_{T_{*} Y}^{\prime} T_{*} X=T_{*}\left(\nabla_{Y} X\right)
$$

Clearly, $\nabla_{Y}^{\prime} X=0$ for $X$ in $\mathscr{X}$. Hence $\nabla^{\prime}=\nabla$.

LeMMA 5. Let $\mathscr{X}$ be a finite-dimensional linear space of vector fields on the compact differentiable manifold $M$. Let $V$ be a fixed vector field on $M$ and let $\mathscr{T}=\left\{T_{\iota}: t \in \boldsymbol{R}\right\}$ be the flow on $M$ corresponding to $V$. For each vector field $X$ on $M$, let $L X=[V, X]$. Then $\mathscr{X}$ is invariant under $\mathscr{T}$ if and only if $L(\mathscr{X}) \subseteq \mathscr{X}$. If $\mathscr{X}$ is so invariant, then

$$
T_{t^{*}}=e^{-t L}
$$

on $\mathscr{X}$. 
Proof. It is known [3, p. 15] that

$$
-L=\lim _{t \rightarrow 0} \frac{1}{t}\left(T_{t^{\bullet}}-I\right)
$$

pointwise, on the space of vector fields, and that $L$ commutes with each $T_{t^{*}}$.

If $\mathscr{X}$ is invariant under $\mathscr{T}$, the conclusion is clear.

Suppose now that $L(\mathscr{X}) \subseteq \mathscr{X}$. Each space $T_{t^{*}}(\mathscr{X})$ is invariant under $L$. Let $\mathscr{Y}$ be the linear span of the union of these spaces. Each point in $\mathscr{Y}$ is contained in a finite-dimensional subspace of $\mathscr{Y}$ invariant under $L$. Define operators $K_{t}$ on $\mathscr{Y}$ by $K_{t}=T_{t} \cdot e^{t L}$. It is readily shown that the derivative of the mapping $t \mapsto K_{t}$ is 0 on $\boldsymbol{R}$. Thus $K_{t}$ is constant in $t$ and equals the identity transformation on $\mathscr{Y}$.

Proof of Theorem 1. Conclusions (I) and (II) have been verified.

(III) Let $\mathscr{Y}$ be as given. Let $\mathscr{E}$ be the space of linear mappings $f: \mathscr{Y} \rightarrow \mathscr{X}$. For each $T$ in $\mathscr{T}$, define $L_{T}: \mathscr{E} \rightarrow \mathscr{E}$ by

$$
\left(L_{T} f\right)=T_{*} f\left(T_{*}\right)^{-1}=T_{*} f T_{-*} .
$$

Clearly, each $L_{T}$ is linear, and $\mathscr{L}=\left\{L_{T}: T \in \mathscr{T}\right\}$ is an abelian group of linear transformations of $\mathscr{E}$. For each $p$ in $M$, define $f_{p}: \mathscr{Y} \rightarrow \mathscr{X}$ by requiring that $f_{p}(Y)$ be that element of $\mathscr{X}$ which agrees with $Y$ at $p$. That is, $\left(f_{p} Y\right)_{p}=Y_{p}$. The mapping $p \mapsto f_{p}$ is differentiable. Since $T_{*}\left(X_{p}\right)=\left(T_{*} X\right)_{T p}$, we have

$$
\begin{aligned}
{\left[\left(L_{T} f_{p}\right) Y\right]_{T p} } & =T_{*}\left[\left(f_{p} T_{-*} Y\right)_{p}\right]=T_{*}\left[\left(T_{-*} Y\right)_{p}\right] \\
& =Y_{T p}=\left(f_{T p} Y\right)_{T p} .
\end{aligned}
$$

Thus, $L_{T} f_{p}=f_{T p}$. The set $\left\{f_{p}: p \in M\right\}$ is invariant under $\mathscr{L}$ and, by Lemma 1 , it must consist of but one point. Therefore, $\mathscr{Y} \subseteq \mathscr{X}$.

(IV) The set $\{[X, Y]: X, Y \in \mathscr{X}\}$ is invariant under $\mathscr{T}$. Its linear span is finitedimensional and invariant under $\mathscr{T}$. By (III), the set is contained in $\mathscr{X}$.

(V) Let $G$ be a simply connected Lie group with Lie algebra isomorphic to $\mathscr{X}$. It is well known that there exists a transitive action, $(x, p) \mapsto x \cdot p$, of $G$ on $M$ which leaves $\mathscr{X}$ invariant. Since $G$ has dimension $n$, there exists a discrete uniform subgroup $\Gamma$ of $G$ and a diffeomorphism $U: G / \Gamma \rightarrow M$ such that

$$
U(x y \Gamma)=x \cdot U(y \Gamma) .
$$

Let $\mathscr{Y}$ be the image under $\pi_{*}$ of the space of right-invariant vector fields on $G$. Since $\mathscr{Y}$ generates the action of $G$ on $G / \Gamma$ and $\mathscr{X}$ generates the action of $G$ on $M$, $U_{*}(\mathscr{X})=\mathscr{Y}$. By Lemma $3, \omega$ is invariant under the action of $G$ on $M$. Hence, $\eta=\left(U^{-1}\right)^{*} \omega$ is invariant under the action of $G$ on $G / \Gamma$, and $\eta$ must correspond to a translation-invariant $n$-form on $G$.

That the diffeomorphisms $U^{-1} T U$ on $G / \Gamma$ are of the stated form follows from Theorem 2 of the next section.

Proof of Corollary 1. By Lemma 3, there exists an appropriate $n$-form on $M$ and Theorem 1 applies. The transformations are affine by Lemma 4, and the structure is unique by (III) of Theorem 1 . 
III.

COROllary 2. Let $M$ be a compact differentiable manifold of dimension $n, \omega$ an $n$-form on $M, \mathscr{X}$ a finite-dimensional linear space of vector fields on $M$. Let there be given a differentiable action of $\boldsymbol{R}^{m}$ on $M$. Suppose that:

(I) $\omega$ is not identically zero, and is invariant under $\boldsymbol{R}^{m}$;

(II) $\mathscr{X}$ is spanning and invariant under $\boldsymbol{R}^{m}$;

(III) Either (i) $M$ is simply connected, or (ii) the action has a fixed point in $M$. Then there exists a nonconstant differentiable function on $M$ which is invariant.

Proof. Let $\mathscr{T}$ be the group of diffeomorphisms. Let $f$ be a differentiable eigenfunction for $\mathscr{T}$ on $M$. Choose $\alpha_{T}$ in $\boldsymbol{R}$ such that

$$
f T=e^{i \alpha} r f .
$$

If there exists a fixed point, $f T=f$ for all $T$, and $f$ is invariant. If $M$ is simply connected, there exists a differentiable function $g: M \rightarrow \boldsymbol{R}$ such that $f=e^{i g}$; then

$$
e^{i g T}=e^{i\left(g+\alpha_{T}\right)}
$$

and $g T-g$ is constant, hence zero; $f$ is invariant.

Suppose now that there does not exist a nonconstant differentiable invariant function on $M$. Then Theorem 1 applies. Let $\mathscr{Y}$ be the space of vector fields on $M$ corresponding to the action of $\boldsymbol{R}^{m}$. Since $\mathscr{Y}$ is invariant under $\mathscr{T}, \mathscr{Y} \subseteq \mathscr{X}$. Thus the system $(M, \mathscr{T})$ is isomorphic to $(G / \Gamma, \mathscr{S})$ where $\mathscr{S}$ consists of translations, $x \Gamma \mapsto a x \Gamma$. If $\mathscr{T}$ has a fixed point, this is surely a contradiction. If $M$ is simply connected, then $\Gamma=\{e\}$, since $\Gamma$ is the fundamental group of $G / \Gamma$. In this case $(M, \mathscr{T})$ is isomorphic to $(G, A)$ where $G$ is a compact simply connected group and $A$ is a connected abelian subgroup of $G$. Let $\bar{A}$ be the closure of $A$. This is a torus (or just $\{e\}$ ) and hence not equal to $G$. But any differentiable function on $G / \bar{A}$ would determine an invariant function on $G$. This is a contradiction.

THEOREM 2. For $k=1,2$ : Let $G_{k}$ be a simply connected Lie group, $\Gamma_{k}$ a discrete subgroup of $G_{k}$, and $\mathscr{X}_{k}$ the space of vector fields on $G_{k} / \Gamma_{k}$ corresponding to the right-invariant vector fields on $G_{k}$. Let $T: G_{1} / \Gamma_{1} \rightarrow G_{2} / \Gamma_{2}$ be a diffeomorphism and suppose that $T_{*}\left(\mathscr{X}_{1}\right)=\mathscr{X}_{2}$. Then there exists a point $a$ in $G_{2}$ and an isomorphism $\alpha: G_{1} \rightarrow G_{2}$ such that $\alpha\left(G_{1}\right)=G_{2}, \alpha\left(\Gamma_{1}\right)=\Gamma_{2}$, and

$$
T\left(x \Gamma_{1}\right)=a \alpha(x) \Gamma_{2}
$$

for $x$ in $G_{1}$. Moreover, if $a^{\prime}$ and $\alpha^{\prime}$ also have this property, then there exists $\gamma$ in $\Gamma_{2}$ such that $a^{\prime}=a \gamma$ and $\alpha^{\prime}(x)=\gamma^{-1} \alpha(x) \gamma$.

Proof. Let $\mathscr{Y}_{k}$ be the space of right invariant vector fields on $G_{k}$. Let $\pi_{k}: G_{k} \rightarrow$ $G_{k} / \Gamma_{k}$ be the natural projection. Then $\mathscr{X}_{k}=\pi_{k^{*}}\left(\mathscr{Y}_{k}\right)$. Thus $\mathscr{Y}_{1}$ and $\mathscr{Y}_{2}$ are isomorphic as Lie algebras, and $T_{*}$ lifts to an isomorphism $L: \mathscr{Y}_{1} \rightarrow \mathscr{Y}_{2}$ such that $\pi_{2^{*}} L=T_{*} \pi_{1^{*}}$. Since $G_{1}$ and $G_{2}$ are simply connected, there exists an isomorphism $\alpha$ of $G_{1}$ onto 
$G_{2}$ such that $L=\alpha_{*}$. Let $e_{k}$ be the identity element of $G_{k}$. Choose $a$ in $G_{2}$ such that $T\left(e_{1} \Gamma_{1}\right)=a \Gamma_{2}$. The two mappings of $G_{1}$ onto $G_{2} / \Gamma_{2}$ given by

$$
x \mapsto T\left(x \Gamma_{1}\right), \quad x \mapsto a \alpha(x) \Gamma_{2},
$$

are equal at $e_{1}$ and have equal differentials. Hence, these two mappings are equal. It is clear that $\alpha\left(\Gamma_{1}\right)=\Gamma_{2}$.

Now suppose that $a^{\prime}$ and $\alpha^{\prime}$ are such that

$$
a^{\prime} \alpha^{\prime}(x) \Gamma_{2}=a \alpha(x) \Gamma_{2}
$$

for all $x$ in $G_{1}$. Letting $x=e_{1}$ shows that $a^{\prime} \Gamma_{2}=a \Gamma_{2}$. There exists $\gamma$ in $\Gamma_{2}$ such that $a^{\prime}=a \gamma$. Thus

$$
\alpha^{\prime}(x) \Gamma_{2}=\gamma^{-1} \alpha(x) \Gamma_{2}=\gamma^{-1} \alpha(x) \gamma \Gamma_{2} .
$$

The two isomorphisms, $x \mapsto \alpha^{\prime}(x)$ and $x \mapsto \gamma^{-1} \alpha(x) \gamma$, are equal on a neighborhood of $e_{1}$ in $G_{1}$. Thus they are equal on $G_{1}$.

EXAMPLE 1. Let $C$ denote the unit circle. There exists a two-dimensional spanning space of vector fields on $C$ and a flow $\mathscr{T}=\left\{T_{t}: t \in R\right\}$ on $C$ such that:

(I) The only invariant Borel measures on $C$ are concentrated on the set $\{1,-1\}$;

(II) $\mathscr{X}$ is invariant under $\mathscr{T}$;

(III) There exist no nonconstant continuous eigenfunctions for $\mathscr{T}$ on $C$.

To see this, let $V$ denote the vector field $d / d \theta$ on $C$, with respect to the parametrization $\theta \mapsto e^{i \theta}$. Define the functions $x, y, z$ on $C$ by

$$
x(p)=\frac{1}{2}(p+\bar{p}), \quad y(p)=(1 / 2 i)(p-\bar{p}), \quad z(p)=p .
$$

Set $Y=y V$ and let $\mathscr{T}=\left\{T_{t}: t \in R\right\}$ be the flow corresponding to the vector field $Y$. By considering at which points $Y$ is positive, negative, or zero, one sees that:

$$
T_{t} 1=1, \quad T_{t}(-1)=-1
$$

for all $t$; and

$$
\lim _{t \rightarrow \infty} T_{t} p=-1, \quad \lim _{t \rightarrow-\infty} T_{t} p=1
$$

for $p$ not in $\{1,-1\}$. Conditions (I) and (III) are thus satisfied.

Define vector fields $X_{1}$ and $X_{2}$ on $C$ by

$$
X_{1}=(1+x) V, \quad X_{2}=(1-x) V .
$$

It is readily seen that

$$
\begin{aligned}
V x & =-y, & V y & =x \\
{[Y, V] } & =-x V, & {[Y, x V] } & =-V \\
{\left[Y, X_{1}\right] } & =-X_{1}, & {\left[Y, X_{2}\right] } & =X_{2} .
\end{aligned}
$$

Let $\mathscr{X}$ be the linear span of $\left\{X_{1}, X_{2}\right\}$. Certainly $\mathscr{X}$ is spanning and (by Lemma 5) invariant under the flow. Thus (II) is satisfied. 
Note that $Y$ is real-analytic on $C$. Thus each $T_{t}$ is a fractional linear transformation leaving 1 and -1 fixed. Such transformations are of the form

$$
p \mapsto(p-a) /(1-a p)
$$

for $a$ real and not equal to \pm 1 . In our case, $-1<a<1$. The system $(C, \mathscr{T})$ is thus isomorphic to a natural action on $G / H$, where $G$ is the group of all fractional linear transformations, and $H$ is not discrete.

Example 2. Let $C$ denote the unit circle. There exists a simply spanning space of vector fields $\mathscr{X}$ on the two-dimensional torus $C \times C$ which is invariant under all translations, but is not a Lie algebra.

To see this, define functions $u$ and $v$ on $C \times C$ by

$$
u(p, q)=\frac{1}{2}(p+\bar{p}), \quad v(p, q)=(1 / 2 i)(p-\bar{p}) .
$$

Define the vector fields $U$ and $V$ on $C \times C$ by $U=\partial / \partial \alpha$ and $V=\partial / \partial \beta$, with respect to the parametrization

$$
(\alpha, \beta) \mapsto\left(e^{i \alpha}, e^{i \beta}\right) .
$$

Now define $X_{1}$ and $X_{2}$ on $C \times C$ by

$$
X_{1}=u U+v V, \quad X_{2}=v U-u V .
$$

One can readily see that

$$
\begin{gathered}
{\left[U, X_{1}\right]=-X_{2}, \quad\left[U, X_{2}\right]=X_{1}} \\
{\left[V, X_{1}\right]=0, \quad\left[V, X_{2}\right]=0} \\
{\left[X_{1}, X_{2}\right]=U .}
\end{gathered}
$$

Let $\mathscr{X}$ be the linear span of $\left\{X_{1}, X_{2}\right\}$. This is simply spanning. It is not a Lie algebra. Moreover, by Lemma $5, \mathscr{X}$ is invariant under flows corresponding to vector fields of the form $\alpha U+\beta V$. But these are just the flows of translations.

\section{REFERENCES}

1. L. Auslander, L. Green, F. Hahn et al., Flows on homogeneous spaces, Princeton Univ. Press, Princeton, N. J., 1963.

2. A. Avez, "Anosov diffeomorphisms", in Topological dynamics, edited by J. Auslander and W. Gottschalk, Benjamin, New York, 1968, pp. 17-51.

3. S. Kobayashi and K. Nomizu, Foundations of differential geometry, Vol. I, Interscience, New York, 1963.

4. R. Palais, A global formulation of the Lie theory of transformation groups, Mem. Amer. Math. Soc., No. 22 (1957).

5. S. Smale, Differentiable dynamical systems, Bull. Amer. Math. Soc. 73 (1967), 747-817.

UNIVERSITY OF WASHINGTON, Seattle, Washington 